\title{
CONTROL STRATEGIES FOR A GANTRY STAGE EQUIPPED WITH FLEXIBLE JOINTS USING FREQUENCY RESPONSE METHODS
}

\author{
P. Poehlmann ${ }^{1 *}$, M. Merx ${ }^{1}$, J. Mueller ${ }^{1}$, S. Ihlenfeldt ${ }^{1,2}$ \\ ${ }^{1}$ Technische Universitaet Dresden, Institute of Mechatronic Engineering, Dresden, Germany \\ ${ }^{2}$ Fraunhofer Institute for Machine Tools and Forming Technology IWU, Dresden, Germany \\ ${ }^{*}$ Corresponding author; e-mail: patrick.poehlmann@tu-dresden.de
}

\begin{abstract}
Gantry stages, consisting of two parallel acting drives, are a well-known example, where a significant interaction between all control systems' in- and outputs is present due to structural coupling. Stability issues and therefore the limitation of the bandwidth of the position control can be a consequence of this effect. The adjustment of the systems mechanical properties using compliant joints, as well as the implementation of centralised control strategies are two possible approaches to overcome these issues and to improve the dynamic and static behaviour of the stage. Frequency domain methods for multivariable systems are used for controller tuning to consider properties like the controls' time delay and high frequency mechanical eigenmodes, which are hard to model otherwise.
\end{abstract}

\section{Keywords:}

gantry stage; linear direct drive; compliant joints; frequency domain

\section{INTRODUCTION}

A high productivity as well as a high product quality are fundamental demands of recent production systems. Accordingly, motion systems of machine tools need to achieve a high control bandwidth, a robust stability behaviour and a low sensitivity to process forces. A wellknown concept, which is able to fulfil these requirements, is the gantry-stage, consisting of two parallel acting drives. In particular, linear direct drives maximise the potential of motion dynamics, since no mechanical transmission is necessary within the drivetrain. However, with the use of two individual drives for one degree of freedom (DOF), the potential of the concept may not be fully exploited. One reason for this is the dynamic interaction of the drives and their control loops, which arises due to the stiff mechanical coupling of the stage.

One way to solve this problem is to increase the DOF of the motion system by unlocking the rotation about the vertical axis (yaw angle). Including compliant joints is an advantageous solution for this purpose. Because of low friction and the absence of backlash, such elements achieve a high accuracy in a small range of motion [Howell 2013]. Consequently, many investigations about the development of high-precision positioning stages with up to six DOFs utilising compliant joints have been published (see e.g. [Kim 2005], [Tian 2010], [Kang 2012]).

Synchronisation strategies using cross coupled (centralised) control on gantry-type motion systems is well known in the state of the art (compare e.g. [Ishizaki 2013] and [Li 2015]). These strategies include a great effort of modelling and parameter identification, since parameterisation and tuning of the controller depends on specific system parameters. To overcome the issue of parameter uncertainties, robust approaches have been developed as well (e.g. [Ma 2019], [Hu 2010]).

In contrast, the usage of frequency response functions (FRFs) offers efficient methods to tune controller gains, without the need for a parameterised model. Various methods for analysing and designing control systems using FRFs are known from the state of the art [Skogestad 2005]. Doyle [Doyle 1981] gives a good overview of strategies to improve the quality of a multivariable control system like the usage of singular values of representative FRF matrices.

Gordon uses loop shaping methods to improve the motion dynamics of a planar (XY) gantry system including an additional active damping function [Gordon 2012]. Peukert [Peukert 2019] uses the Independent Modal Space Control (IMSC) for the active damping of a gantry stage. FRF methods are used for the tuning and the analysis of the control system. In previous work [Poehlmann 2020], gantry stages with varying rotational stiffness were compared and the positive effect of compliant joints regarding stability and control bandwidth was shown while using a classical cascaded control scheme. One drawback of the concept of compliant joints is, that the reduction of the rotational stiffness leads to a higher sensitivity to process loads, since external torque cannot be countered by the linear guides. In this study, compliant joints are combined with a simple centralised decoupling control strategy, to further improve 
the dynamic system behaviour. To show the effect of the system properties in combination with centralised and decentralised control strategies, the stage equipped with compliant joints is compared to the one without those.

In this contribution, frequency domain methods are used (see Section 4) to tune the controllers offline and calculate stability margins as well as closed loop behaviour of the whole system. For this purpose, the Nyquist stability criterion for multivariable systems is applied (see [MacFarlane 1977]). With the use of measured FRFs for the controller design, system properties like time delay and resonance peaks at high frequencies, can be considered. In contrast, this would lead to a notable increase of modelling and tuning effort using parametric approaches. The tuning of a single drive using FRF methods, is already common in industrial applications. In general, an extension to a multivariable approach would offer a robust und widely automated method for controller tuning of mechanically coupled drive arrangements.

\section{EXPERIMENTAL SETUP}

The test rig is depicted in Fig. 1. It consists of ironless linear direct drives (Tecnotion UXX6N) with fixed magnet yokes and absolute linear measurement systems (Heidenhain LIC4117) with an accuracy of $\pm 5 \mu \mathrm{m}$ and a resolution of $1 \mathrm{~nm}$. The entire motion system consists of four drives, which are carried on two guiding rails (Hiwin HG15) with a cross-axial distance of $770 \mathrm{~mm}$. The drives are connected by bridge plates to form two gantry stages. One of them is used in this investigation. The moving parts of the system are mainly made of aluminium to achieve a lightweight design of the gantry $(29.4 \mathrm{~kg})$.

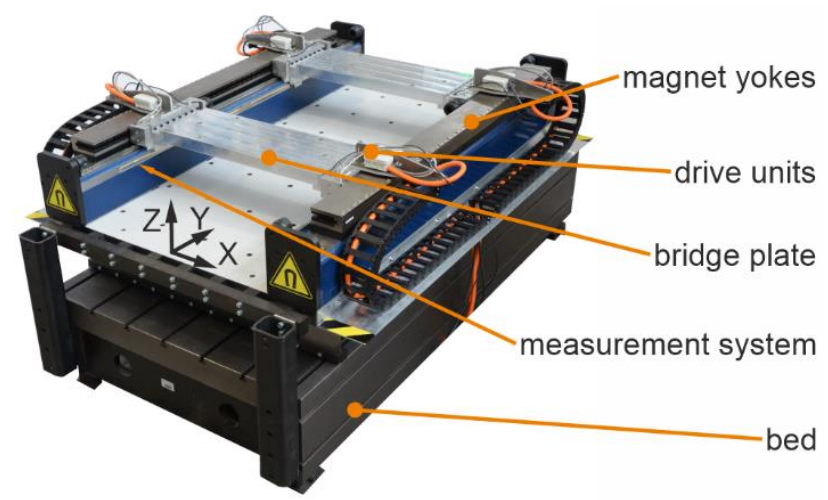

Fig. 1: The test bed.

The test rig is controlled by a PC-based real-time system (TwinCAT3 by Beckhoff). In order to realise centralised control strategies instead of the decentralised cascaded scheme, the drives (AX 5206) are running in current control mode with a cycle time of $62.5 \mu \mathrm{s}$ and the velocity and position control is performed in the real-time system with a cycle time of $125 \mu \mathrm{s}$. An EtherCAT fieldbus connects the drives and the real-time system.

In the following investigations, two mechanical configurations of the gantry stage are compared. In the first configuration, a stiff coupling element connects the bridge plate of the gantry with the drives on both sides (see Fig. 2). Only high forces lead to a notable yaw rotation and there is no usable rotational DOF. Contrary, the stage is insensitive to external torque. This characteristic is generally given in a design with a short cross-axial distance and a high axial distance of the bearings. In the second configuration, the rigid coupling elements are replaced by compliant joints to adjust the mechanical properties of the stage (see Fig. 3).
The compliant joints have a low stiffness corresponding to rotational motion about the vertical axis. This is achieved by the specific arrangement of different flexible link elements, pointing to a common centre. The flexible links are designed as stacks of multiple thin spring steel sheets in order to achieve a low stiffness of the desired DOF while maintaining high stiffness of the other DOFs. As a result, a yaw-rotation $\theta$ can be realised by a relative displacement of the linear drives while only a low force is required. However, a system without joints can also have the characteristics of a small rotational stiffness. This is the case, for example, if the bridge spans over a wide cross-axial distance or has a high moment of inertia. A more detailed description of the designed compliant joints is given in [Poehlmann 2020].
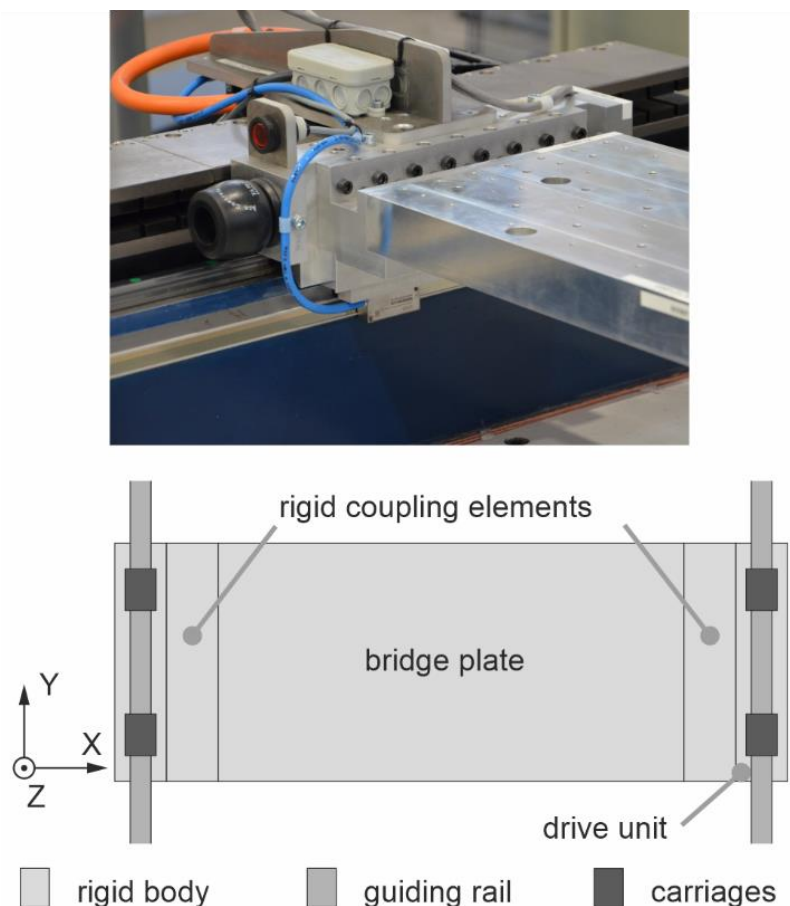

Fig. 2: Gantry stage with rigid coupling elements.

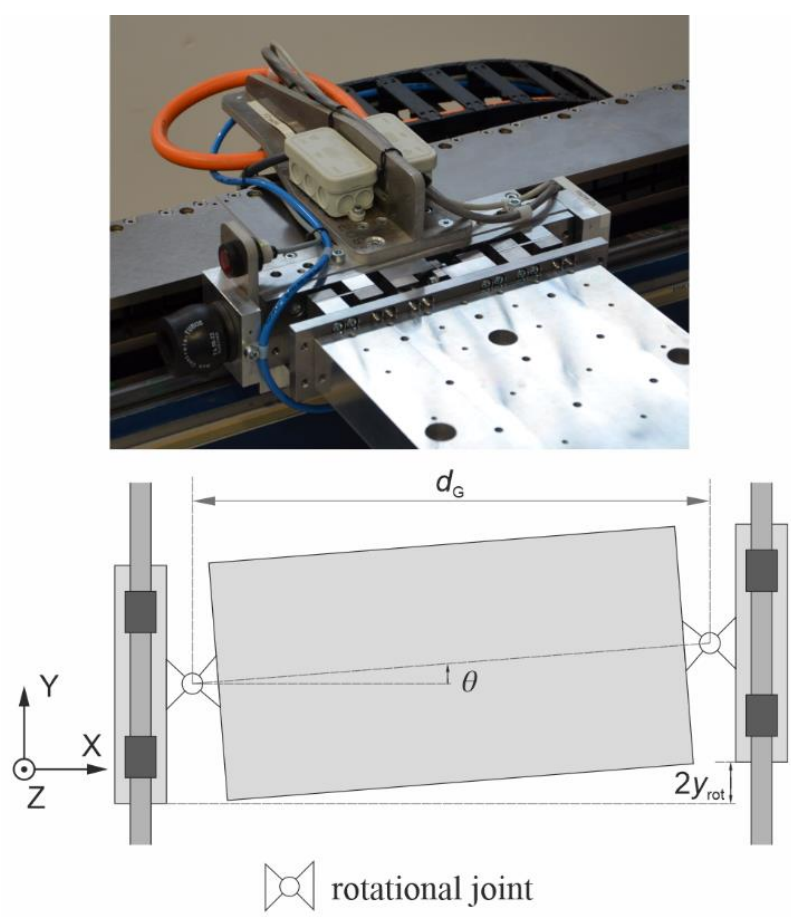

Fig. 3: Gantry stage with compliant coupling elements. 


\subsection{System Identification}

The nonparametric model of the system in the frequency domain is given by

$\left(\begin{array}{l}v_{1}(\mathrm{j} \omega) \\ v_{2}(\mathrm{j} \omega)\end{array}\right)=\left(\begin{array}{ll}G_{11}(\mathrm{j} \omega) & G_{12}(\mathrm{j} \omega) \\ G_{21}(\mathrm{j} \omega) & G_{22}(\mathrm{j} \omega)\end{array}\right) \cdot\left(\begin{array}{l}f_{1}(\mathrm{j} \omega)+d_{1}(\mathrm{j} \omega) \\ f_{2}(\mathrm{j} \omega)+d_{2}(\mathrm{j} \omega)\end{array}\right)$,

$\boldsymbol{v}(\mathrm{j} \omega)=\boldsymbol{G}(\mathrm{j} \omega) \cdot(\boldsymbol{f}(\mathrm{j} \omega)+\boldsymbol{d}(\mathrm{j} \omega))$,

with the vectors of input forces $\boldsymbol{f}(\mathrm{j} \omega)$, the disturbances $\boldsymbol{d}(\mathrm{j} \omega)$, the output velocities $\boldsymbol{v}(\mathrm{j} \omega)$ and the two-by-two matrix of FRFs $\boldsymbol{G}(\mathrm{j} \omega)$. The velocities are obtained by the derivation of the linear encoder signals in the drive units. The forces are obtained by multiplying the motor current with the nominal force constant of $124 \mathrm{~N} / \mathrm{A}$.

The system is excited by only one drive at a time, using pseudo random binary sequence (PRBS) signals fed through a low pass filter (time constant: $T=1 /(1000 \pi) \mathrm{s}$ ). The FRFs of the Matrix $\boldsymbol{G}(\mathrm{j} \omega)$ are then calculated by correlation analysis (see e.g. [Bohn 2016]). In the identification experiment, cascaded velocity and position controllers with a low bandwidth (achieved by low gains) are used to keep the stage in continues motion to avoid issues like stick-slip arising from static friction.
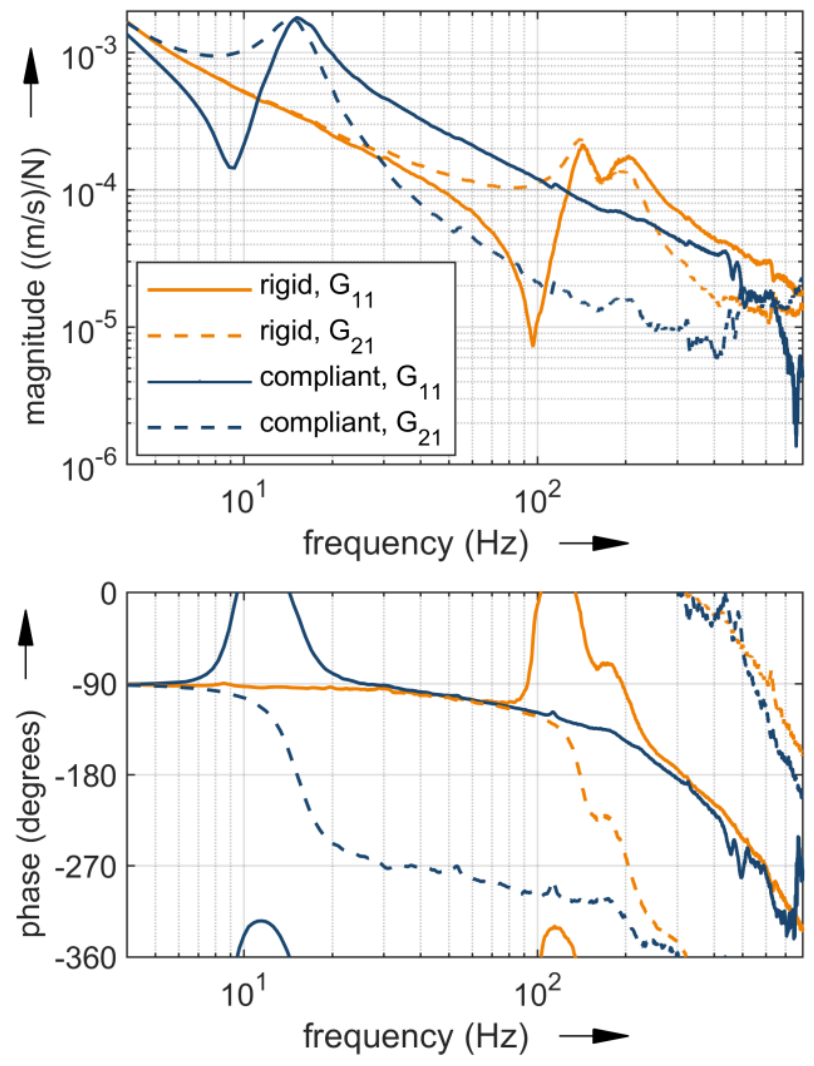

Fig. 4: System FRFs of both configurations.

Fig. 4 shows the resulting FRFs of the rigid and the compliant configuration. Due to the symmetry of the system, the illustration of $G_{12}(\mathrm{j} \omega)$ and $G_{22}(\mathrm{j} \omega)$ is omitted. Because of the integral character of the rigid body motion, each FRF consists of a decreasing amplitude with a ratio of 0.1 per frequency decade and a phase of $-90^{\circ}$ at low frequencies. The first and highest resonance peak of each system is characterised by the opposite directed motion of the drives. This is indicated by the relative phase shift of $180^{\circ}$ at the corresponding frequency value. With the use of the compliant joints, this first eigenfrequency is shifted from approximately $168 \mathrm{~Hz}$ (compliant configuration) to $16 \mathrm{~Hz}$. Other resonance peaks at higher frequencies result from the dynamic deformation of other mechanical parts like the bridge plate and are not analysed in detail. The increasing phase shift for raising frequencies can be represented by a static time delay of approximately $0.875 \mathrm{~ms}$ for both configurations.

\section{CONTROL STRATEGIES}

In this paper, two control strategies are applied to both the rigid and the compliant configuration. The parameter tuning of both strategies is done iteratively with the use of the matrix of the measured FRFs (see Eq. (1)). If the system is identified once, the expected closed loop characteristics of arbitrary controllers and parameter sets can be calculated. This includes e.g. stability margins or quality criterions like load sensitivity functions. A requirement for this method is the linearity of the system at the operating point. The overall control schemes are depicted in Fig. 5. In addition to the control strategies described in the following sections, a low pass filter with a cut off frequency of $1000 \mathrm{~Hz}$ and individual notch filters (for frequencies above $1000 \mathrm{~Hz}$ ) are used to avoid stability issues at higher frequencies (see "filter" in Fig. 5). The filters are considered to be a part of the system $\boldsymbol{G}(\mathrm{j} \omega)$ and will be omitted in the remainder of this study.

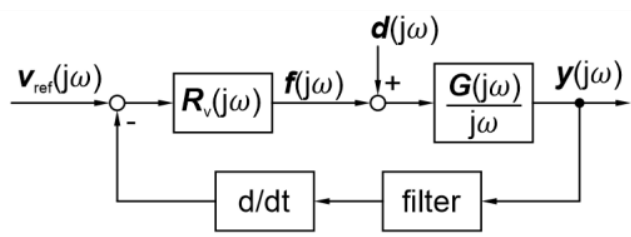

a) velocity control

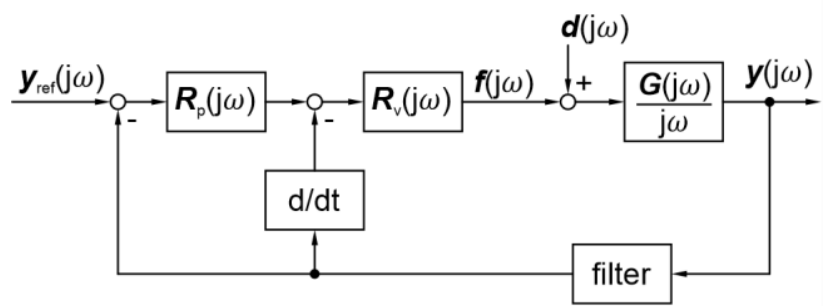

b) cascaded position control

Fig. 5: Schemes of the velocity and position control.

\subsection{Decentralised control strategy}

The first strategy uses a classic decentralised scheme with individual cascaded position $(\mathrm{P})$ and velocity $(\mathrm{PI})$ controllers for both drives. Each of the controllers is set independently by tuning the parameters of the velocity and position control loops one after another. In practice, this strategy is beneficial because the tuning procedure can be used at the machine without any knowledge about the system parameters. However, if there is a strong coupling between the drives (esp. mechanical coupling), the parameter set of one control loop will affect the stability margin of the other and an acceptable closed loop behaviour may be difficult to be found. Since the system in this example is mainly symmetric, the gains are set equally to the controllers of both drives. The procedure of the decentralised strategy starts with the tuning of the velocity loop gain $K_{\mathrm{v}}$, while $K_{\mathrm{I}}$ and $K_{\mathrm{p}}$ are set to zero. The control law of the decentralised velocity controller can be written as:

$\boldsymbol{R}_{\mathrm{v}, \mathrm{DC}}(\mathrm{j} \omega)=\left(\begin{array}{cc}K_{\mathrm{V}} & 0 \\ 0 & K_{\mathrm{v}}\end{array}\right)+\left(\begin{array}{cc}K_{\mathrm{I}} & 0 \\ 0 & K_{\mathrm{I}}\end{array}\right) \cdot \frac{1}{\mathrm{j} \omega}$,

where $K_{\mathrm{I}}=K_{\mathrm{v}} / T_{\mathrm{N}}$. The gain $K_{\mathrm{v}}$ is raised up until the critical value is reached where the output starts to oscillate. This point can be detected by the use of the eigenfunctions of 
the open loop, shown in Section 4.1. To provide adequate gain margins, the critical gain value is divided by two. The time constant $T_{\mathrm{N}}$ is set to $10 \mathrm{~ms}$ in each case. This parameter is not varied in this paper, to keep the different configurations and control strategies comparable. In the last step, the position control is added to the closed velocity loop:

$\boldsymbol{R}_{\mathrm{p}, \mathrm{DC}}(\mathrm{j} \omega)=\left(\begin{array}{cc}K_{\mathrm{p}} & 0 \\ 0 & K_{\mathrm{p}}\end{array}\right)$

In the tuning procedure, the gain $K_{\mathrm{p}}$ is raised until the response magnitude of the closed loop slightly hits the value of 1 with no overshoot. Section 4.2 describes this aspect in detail.

\subsection{Centralised control strategy}

The second control strategy uses transformation matrices

$\boldsymbol{T}_{\mathrm{y}}=\left(\begin{array}{cc}0.5 & 0.5 \\ 0.5 & -0.5\end{array}\right)$ and $\boldsymbol{T}_{\mathrm{f}}=\left(\begin{array}{cc}1 & 1 \\ 1 & -1\end{array}\right)$,

to split the system in a translational and a rotational DOF:

$\left(\begin{array}{l}y_{\text {tra }} \\ y_{\text {rot }}\end{array}\right)=\left(\begin{array}{cc}0.5 & 0.5 \\ 0.5 & -0.5\end{array}\right)\left(\begin{array}{l}y_{1} \\ y_{2}\end{array}\right)=\boldsymbol{T}_{\mathrm{y}} \cdot \boldsymbol{y}$.

Both the translational and the rotational DOF are controlled with a cascaded velocity and position controller, similar to the decentralised strategy. A main advantage of the new coordinates $y_{\text {trans }}$ and $y_{\text {rot }}$ is that the two controllers can be tuned independently and individual gains are used for the translational and rotational motion. The tuning of each of the controllers is done with the same procedure as in the decentralised case. First, the gain $K_{\mathrm{v}}$ of the velocity loop is raised to the critical value, while integral gain and position control are inactive. Subsequently, the time constant $T_{\mathrm{N}}$ is set to a value of $10 \mathrm{~ms}$. The control law of the velocity controller is given by:

$\boldsymbol{R}_{\mathrm{v}, \mathrm{CC}}=\boldsymbol{T}_{\mathrm{f}} \cdot\left(\left(\begin{array}{cc}K_{\mathrm{v}, \text { tra }} & 0 \\ 0 & K_{\mathrm{v}, \text { rot }}\end{array}\right)+\left(\begin{array}{cc}K_{\mathrm{I}, \text { tra }} & 0 \\ 0 & K_{\mathrm{I}, \text { rot }}\end{array}\right) \cdot \frac{1}{\mathrm{j} \omega}\right) \cdot \boldsymbol{T}_{\mathrm{y} \cdot}(6)$

Finally, the position controller

$\boldsymbol{R}_{\mathrm{p}, \mathrm{CC}}=\boldsymbol{T}_{\mathrm{f}} \cdot\left(\begin{array}{cc}K_{\mathrm{p}, \text { tra }} & 0 \\ 0 & K_{\mathrm{p}, \mathrm{rot}}\end{array}\right) \cdot \boldsymbol{T}_{\mathrm{y}}$

is tuned to push the response function of the closed position loop as close as possible to the value of 1 without passing it. This is done with two different response functions corresponding to a translational and a rotational reference value (see Section 4.2).

\section{RESULTS}

\subsection{Velocity control}

Both strategies are based on cascaded control schemes. The position control is turned off to analyse the velocity control in the first step. The controller gains are tuned using the procedures described in Section 3. The stability margins of the system are found with the Nyquist stability criterion. In case of the multivariable system, the criterion is applied to the eigenvalues of the open velocity loop FRF matrix

$\lambda_{i}(\mathrm{j} \omega)=\operatorname{eig}\left(\boldsymbol{G}(\mathrm{j} \omega) \cdot \boldsymbol{R}_{\mathrm{v}}(\mathrm{j} \omega)\right)=\operatorname{eig}\left(\boldsymbol{G} \boldsymbol{R}_{\mathrm{v}}(\mathrm{j} \omega)\right)$,

which are functions of frequency as well (see [MacFarlane 1977]). In this example, the gain of the velocity loop $K_{\mathrm{P}}$ is raised until one of the functions $\lambda_{i}(\mathrm{j} \omega)$ reaches the magnitude value of 1 at the frequency where a phase shift of $-180^{\circ}$ is present. The parameters that are found for all mechanical configurations and control strategies are listed in Tab. 1 (half value of the critical $K_{\mathrm{v}}$ is used).

Fig. 6 and Fig. 7 depict the magnitude and phase of the eigenvalues resulting from the open velocity loop with the given $K_{\mathrm{v}}$ and $T_{\mathrm{N}}$. The crossover frequencies, where the phase is equal to a value of $-180^{\circ}$, are marked with vertical dashed lines. In this work the difference between the magnitude of the largest eigenvalue and the magnitude value of 1 is specified as the gain margin in this work.

Tab. 1: Gain values of the velocity and position controllers.

\begin{tabular}{ccccc}
\hline $\begin{array}{c}\text { Config- } \\
\text { uration }\end{array}$ & control & $\begin{array}{c}K_{\mathrm{P}} \text { in } \\
\mathrm{A} /(\mathrm{m} / \mathrm{s})\end{array}$ & $\begin{array}{c}T_{\mathrm{N}} \text { in } \\
\mathrm{ms}\end{array}$ & $\begin{array}{c}K_{\mathrm{p}} \text { in } \\
1 / \mathrm{s}\end{array}$ \\
\hline $\begin{array}{c}\text { rigid } \\
\text { compliant }\end{array}$ & $\mathrm{DC}$ & 28.5 & 10.0 & 108.0 \\
rigid & $\mathrm{DC}$ & 58.5 & 10.0 & 200.0 \\
compliant & CC trans. & 75.5 & 10.0 & 250.0 \\
rigid & CC rot. & 24.5 & 10.0 & 470.0 \\
compliant & CC rot. & 55.0 & 10.0 & 247.0 \\
\hline
\end{tabular}

With the tuning strategies described in Section 3, all systems show approximately the same gain margins. The value is slightly smaller than 0.5 , since the shape of the eigenvalues is changed when the integral part (using $T_{\mathrm{N}}$ ) is added to the controllers. Using the decentralised strategy (see Fig. 6), the second eigenvalue $\lambda_{2}(\mathrm{j} \omega)$ (both configurations) shows a lower magnitude than the first eigenvalue $\lambda_{1}(\mathrm{j} \omega)$ at the phase crossover frequency. When the centralised strategy is applied, $\lambda_{1}(\mathrm{j} \omega)$ and $\lambda_{2}(\mathrm{j} \omega)$ of one configuration are nearly equal at this frequency. With the individual tuning of translational and rotational controller, both eigenvalues are shifted close to the same stability margin.
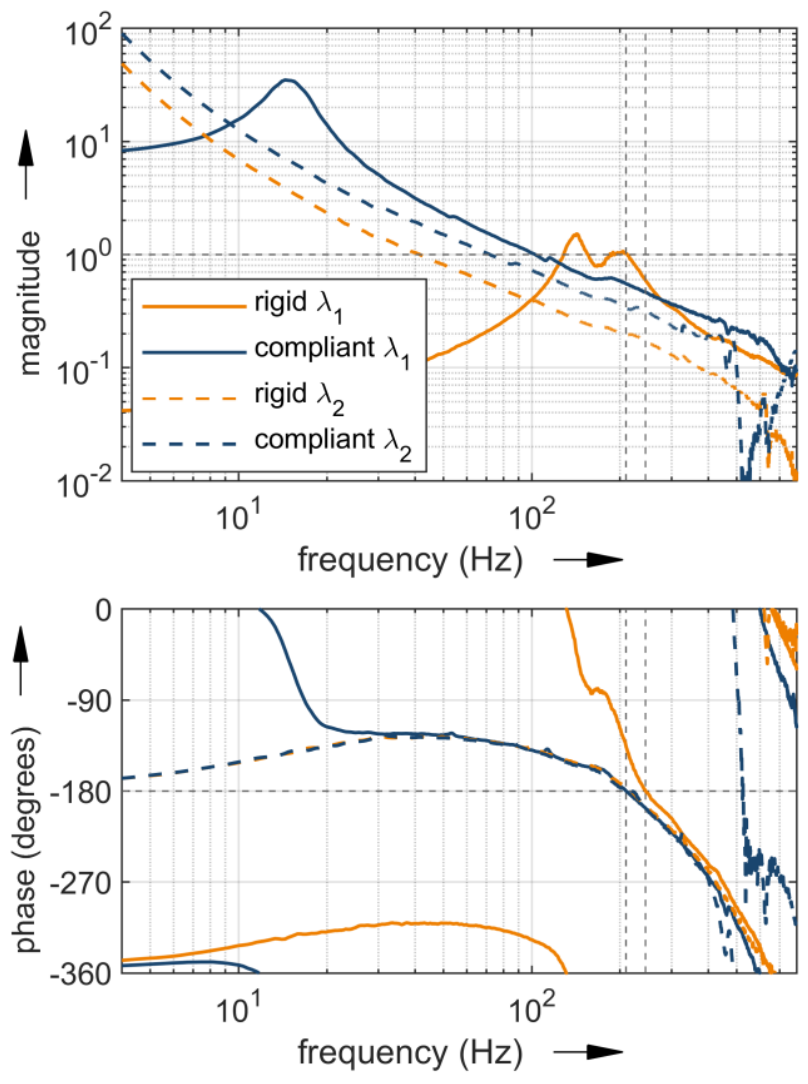

Fig. 6: Open loop eigenvalues of the decentralised control (DC) strategy. 
The gain values given in Tab. 1 show a difference between the rigid and the compliant configuration. If the same contro strategy is used, the resulting gain $K_{\mathrm{v}}$ of the compliant configuration is always higher than the corresponding gain of the rigid configuration. The reason is the resonance peak of the mechanical eigenfrequency, which reduces the gain margin at the phase crossover frequency of the eigenvalue. With compliant elements, the first mechanical eigenfrequency is shifted to a lower value, where $\lambda_{1}(j \omega)$ is dominated by higher magnitudes and phase margins (see [Poehlmann 2020]).
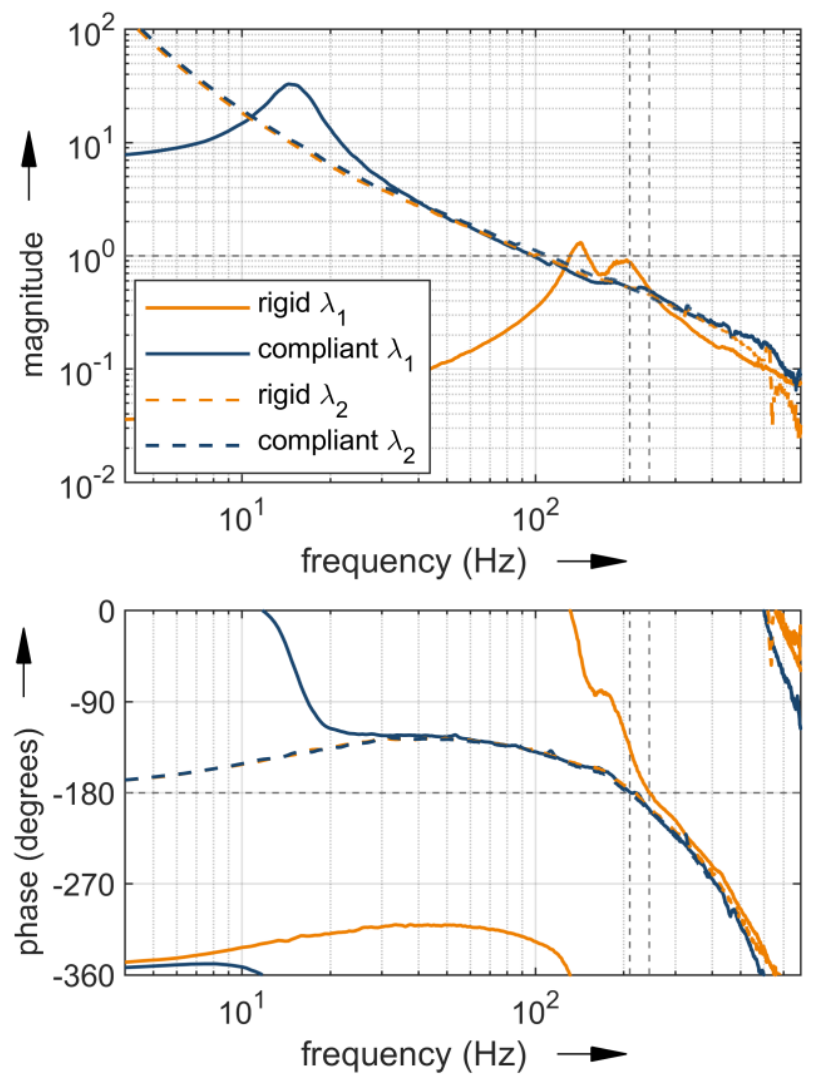

Fig. 7: Open loop eigenvalues of the centralised control (CC) strategy.

The transfer behaviour of the closed velocity loop is given by the matrix $\boldsymbol{G}_{\mathrm{v}}(\mathrm{j} \omega)$ :

$$
\begin{aligned}
& \boldsymbol{v}(\mathrm{j} \omega)=\boldsymbol{G}_{\mathrm{v}}(\mathrm{j} \omega) \boldsymbol{v}_{\mathrm{ref}}(\mathrm{j} \omega) \\
& \boldsymbol{G}_{\mathrm{v}}(\mathrm{j} \omega)=\left(\mathbf{I}+\boldsymbol{G R}_{\mathrm{v}}(\mathrm{j} \omega)\right)^{-1} \boldsymbol{G}_{\mathrm{v}}(\mathrm{j} \omega) .
\end{aligned}
$$

The transfer functions shown in Fig. 8 represent the average velocity of both drives in case of symmetric reference values (translational motion):

$$
\boldsymbol{G}_{\mathrm{v}, \mathrm{t}}(\mathrm{j} \omega)=\left(\begin{array}{ll}
0.5 & 0.5
\end{array}\right) \cdot \boldsymbol{G}_{\mathrm{v}}(\mathrm{j} \omega) \cdot\left(\begin{array}{l}
1 \\
1
\end{array}\right) \text {. }
$$

To achieve a high accuracy and dynamic of the control system, the FRF needs to be close to the magnitude of 1 in a wide frequency range. In the given example, the masses of both configurations are approximately equal. Using the DC strategy, the behaviour of the translational motion is mainly affected by the controller gain. Therefore the compliant configuration achieves a higher bandwidth, compared to the rigid configuration. Applying the CC strategy, the dynamic behaviour is further improved. In this case, the difference between rigid and compliant configuration is very small, since the joints mainly affect the rotational but not the translational characteristics of the system. Since individual controllers are used for translational and rotational motion (DOFs), the resonance peak of the lowest mechanical eigenfrequency of the rigid configuration has no remarkable effect on the translational control loop.

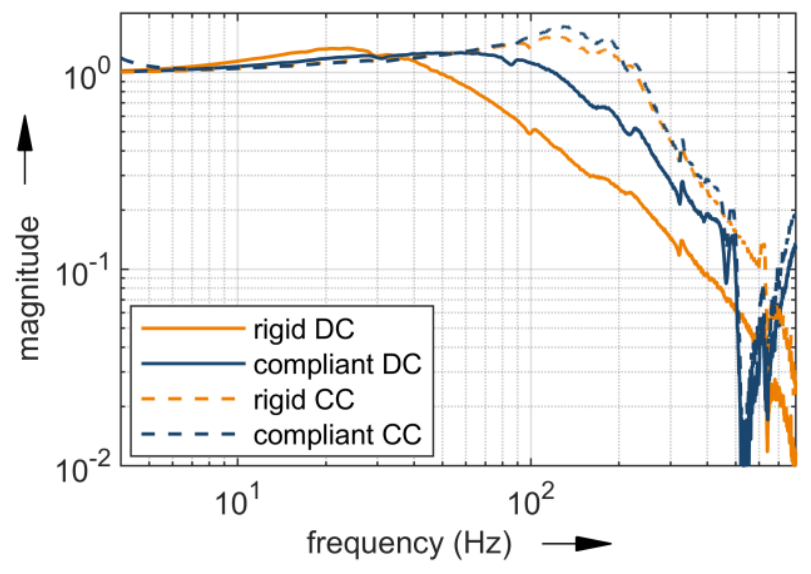

Fig. 8: Magnitudes of the closed loop response of the velocity control to symmetric reference values $\left|G_{\mathrm{v}, \mathrm{t}}(\mathrm{j} \omega)\right|$.

\subsection{Position control}

The position controllers of all configurations and strategies are tuned in consideration of the closed loop FRF corresponding to a translational (symmetric) position reference value given by:

$G_{\mathrm{y}, \mathrm{t}}(\mathrm{j} \omega)=\left(\begin{array}{ll}0.5 & 0.5\end{array}\right) \cdot \boldsymbol{G}_{\mathrm{y}}(\mathrm{j} \omega) \cdot\left(\begin{array}{l}1 \\ 1\end{array}\right)$,

where $\boldsymbol{G}_{\mathrm{y}}(\mathrm{j} \omega)$ is the full response matrix of the closed loop:

$\boldsymbol{y}(\mathrm{j} \omega)=\boldsymbol{G}_{\mathrm{y}}(\mathrm{j} \omega) \boldsymbol{y}_{\mathrm{ref}}(\mathrm{j} \omega)$,

$\boldsymbol{G}_{\mathrm{y}}(\mathrm{j} \omega)=\left(\mathbf{I}+\boldsymbol{G}_{\mathrm{v}} \boldsymbol{R}_{\mathrm{p}}(\mathrm{j} \omega)\right)^{-1} \boldsymbol{G}_{\mathrm{v}} \boldsymbol{R}_{\mathrm{p}}(\mathrm{j} \omega)$.

In case of the centralised control, the response to a rotational reference value

$G_{\mathrm{y}, \mathrm{r}}(\mathrm{j} \omega)=\left(\begin{array}{ll}0.5 & -0.5\end{array}\right) \cdot \boldsymbol{G}_{\mathrm{y}}(\mathrm{j} \omega) \cdot\left(\begin{array}{c}1 \\ -1\end{array}\right)$,

is used additionally, to tune the gain of the rotational controller. Tab. 1 lists the gains $K_{\mathrm{v}}$ of all setups and Fig. 9 displays the magnitudes of the resulting response to a translational reference motion $\left|G_{\mathrm{y}, \mathrm{t}}(\mathrm{j} \omega)\right|$. As a result of the improved bandwidth of the decentralised velocity controller, the bandwidth of the position control is improved as well, if compliant elements are used instead of rigid ones. When the CC strategy is applied, an even larger control bandwidth is achievable and, in analogy with the velocity control, there is only a negligible difference between the two mechanical configurations.

Fig. 10 depicts the closed loop response to a rotational reference motion according to (15). Using the rigid configuration and the classical control scheme (DC) the rotation cannot be controlled. This is indicated by the magnitude of the FRF, which is far below the value of 1 in the full frequency range. Using the centralised control strategy, the magnitude is raised, but does not lead to an acceptable transfer function. This is a result of the high rotational stiffness of the bridge, which acts against the actuators. However, this is no issue, if a rotational DOF is not required. In this case, the deactivation of the rotational controller can be advantageous to reduce the actuator forces and power consumption. 


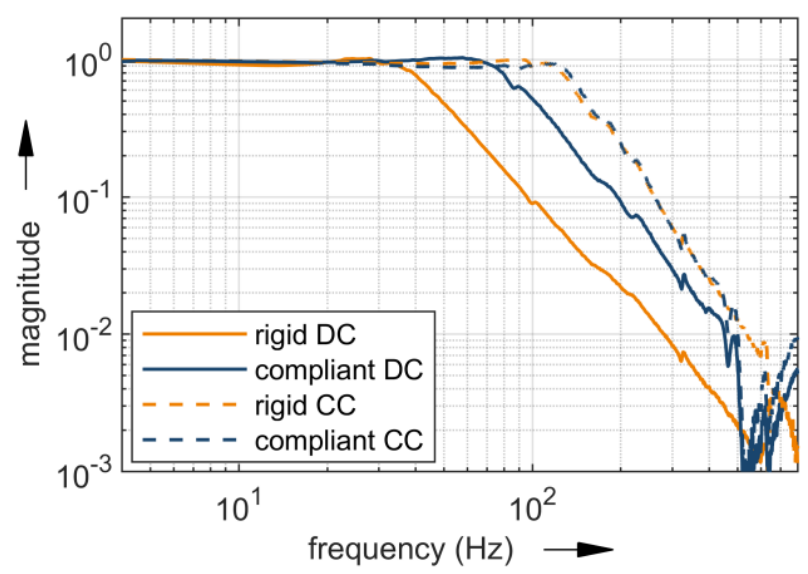

Fig. 9: Closed loop response of the position control to symmetric reference values $\left|G_{\mathrm{y}, \mathrm{t}}(\mathrm{j} \omega)\right|$.

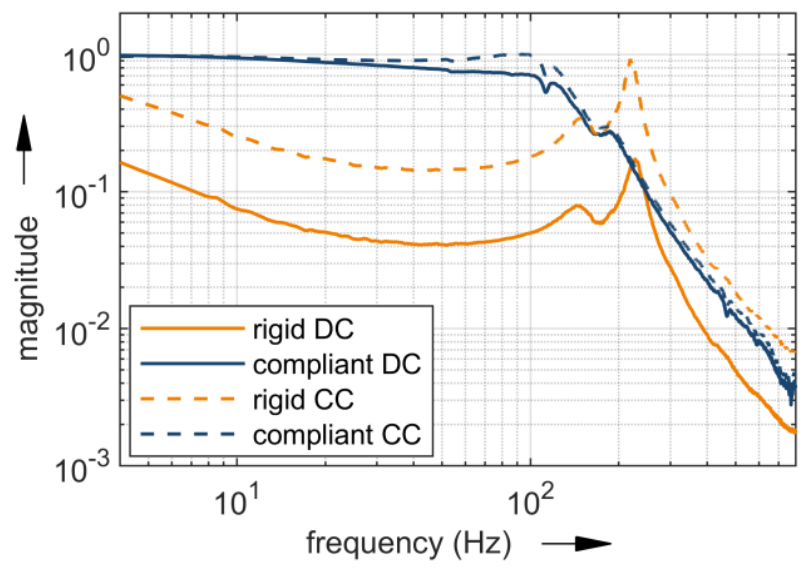

Fig. 10: Closed loop response of the position control $\left|G_{\mathrm{y}, \mathrm{r}}(\mathrm{j} \omega)\right|$ to a rotational reference motion.

\subsection{Load Response}

This section addresses the sensitivity of the different systems to external loads. The matrix $\boldsymbol{G}_{\mathrm{d}}(\mathrm{j} \omega)$ represents the response of the output to a disturbance vector $\boldsymbol{d}(\mathrm{j} \omega)$ :

$\boldsymbol{y}(\mathrm{j} \omega)=\boldsymbol{G}_{\mathrm{d}}(\mathrm{j} \omega) \boldsymbol{d}(\mathrm{j} \omega)=\boldsymbol{G}_{\mathrm{d}}(\mathrm{j} \omega)\left(\begin{array}{l}d_{1}(\mathrm{j} \omega) \\ d_{2}(\mathrm{j} \omega)\end{array}\right)$.

Here $d_{1}$ and $d_{2}$ are assumed to be loads that act in the same way as the forces of the actuators. In analogy with the previous sections, the matrix $\boldsymbol{G}_{\mathrm{d}}$ is split into a translational motion response to a symmetric load $\left(d_{1}=d_{2}\right)$ :

$G_{\mathrm{d}, \mathrm{t}}(\mathrm{j} \omega)=\left(\begin{array}{ll}0.5 & 0.5\end{array}\right) \cdot \boldsymbol{G}_{\mathrm{d}}(\mathrm{j} \omega) \cdot\left(\begin{array}{l}1 \\ 1\end{array}\right)$,

as well as a rotational response to opposite directed loads $\left(d_{1}=-d_{2}\right)$ :

$G_{\mathrm{d}, \mathrm{r}}(\mathrm{j} \omega)=\left(\begin{array}{ll}0.5 & -0.5\end{array}\right) \cdot G_{\mathrm{d}}(\mathrm{j} \omega) \cdot\left(\begin{array}{c}1 \\ -1\end{array}\right)$.

The magnitudes $\left|G_{\mathrm{d}, \mathrm{t}}(\mathrm{j} \omega)\right|$ of the different systems are illustrated in Fig. 11. The graphs show a reduction of the load sensitivity when higher gains are used in the controllers. A load that acts mainly in the direction of the motion axis has to be countered by the drives and $G_{\mathrm{d}, \mathrm{t}}(\mathrm{j} \omega)$, as a consequence, is mainly affected by the performance of the control. The magnitudes of $G_{\mathrm{d}, \mathrm{r}}(\mathrm{j} \omega)$, which indicate the sensitivity to opposite directed loads (equivalent to a torque), show the disadvantage of the compliant configuration (see Fig. 12). Torque, which is acting on the bridge, have to be countered by the drives. This leads to a higher load sensitivity (DC and CC) in the lower frequency range $(<100 \mathrm{~Hz})$ compared to the rigid configuration where the torque act against the linear guides. Consequently, this load scenario will lead to a higher power consumption when the compliant configuration is used. On the contrary, the rigid configuration (both $C D$ and $C C$ ) show a significant raise of the magnitude at a frequency of about $220 \mathrm{~Hz}$, which is not present in the compliant configuration. Even if the magnitude of the load response is lower in the rest of the frequency range, this resonance peak can lead to stability issues in an application, especially like milling.

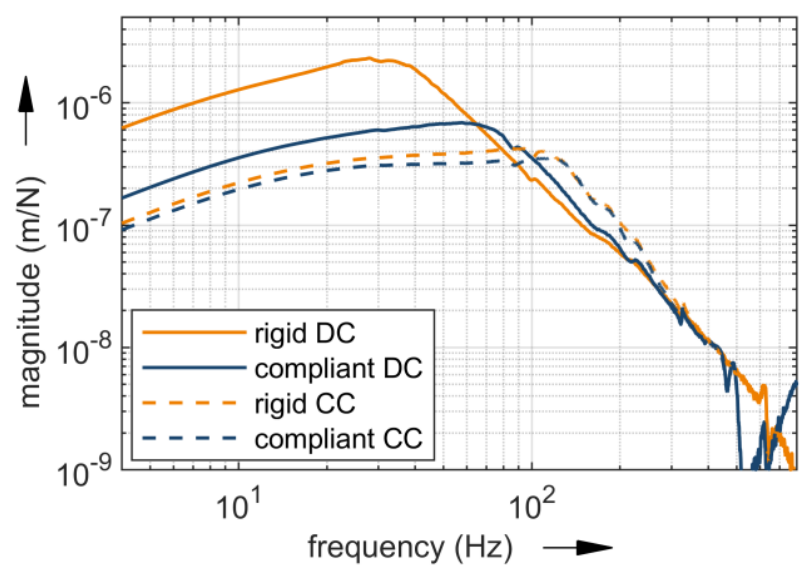

Fig. 11: Translational load response $\left|G_{\mathrm{d}, \mathrm{t}}(\mathrm{j} \omega)\right|$.

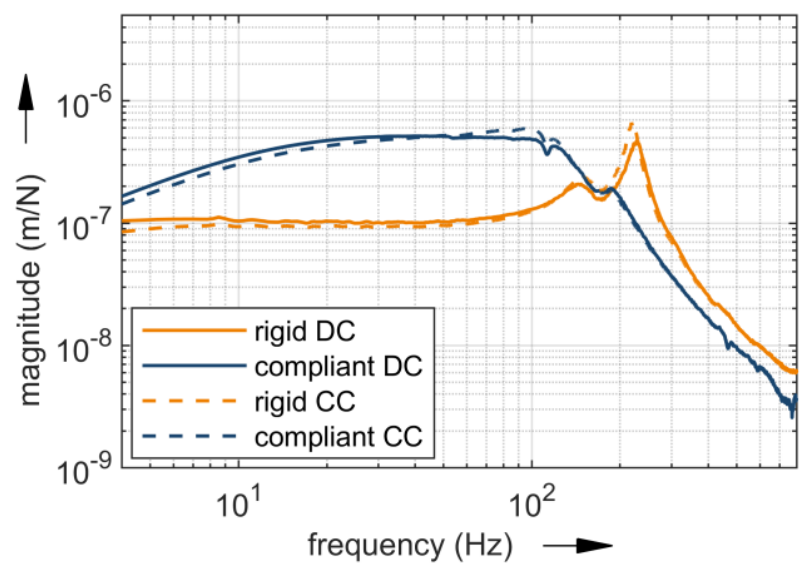

Fig. 12: Rotational response to opposite directed loads (torque) $\left|G_{\mathrm{d}, \mathrm{r}}(\mathrm{j} \omega)\right|$.

\section{SUMMARY}

This paper presents experimental investigations of a gantry stage driven by linear direct drives. In the standard configuration, the bridge of the stage is connected with the two drives by rigid elements. It is shown, that the control bandwidth of the commonly used decentralised controllers (DCs) is reduced, if the first mechanical eigenfrequency is close to the phase crossover frequency of the open loop eigenvalues. To improve the dynamic behaviour of the stage, the rigid coupling elements are replaced by compliant joints, which lead to a reduction of the first eigenfrequency and as a consequence, to higher gain margins. Furthermore, a centralised control strategy with a static transformation of the position and force values (CC) is applied to both configurations as an alternative to the decentralised strategy. All controllers are implemented utilising hardware, which is commonly used in industrial applications. The design of the motion stage in this example 
is simple. In a more practical case, where more DOFs are included, the transformation of the centralised control needs to be adapted to the variable structure.

Both the replacement of the rigid elements and the usage of the decentralised control strategy lead to an improvement of the resulting control bandwidth of the motion stage. The decision which of the concepts should be used, depends on more aspects, like the question if a rotational DOF is required (e.g. to compensate angular errors of the bridge) or if a centralised control strategy can be realised with the available hardware.

If the rotational DOF is not necessary and the rigid configuration is used, it is possible to use the CC strategy to improve the dynamic behaviour of the translational motion. Due to the high rotational stiffness of the rigid configuration, the rotational controller can be deactivated to reduce the occurring forces and power consumption.

The combination of the compliant configuration and the centralised control strategy results in the largest motion bandwidth of both the translational and the rotational DOF. The purpose of the tuning procedure, exemplary used in this investigation, is to obtain comparable results for the different strategies. The procedure can be adapted to meet further demands, like a reduction of the gain values to improve stability margins, if a lower control bandwidth is sufficient. Another possibility is the optimisation of the disturbance response of the system.

Measured FRFs are used to analyse the characteristics of the multivariable systems and the control strategies. Using these methods, parameter identification is not necessary and no additional measurement systems are required. Therefore, frequency domain methods for multivariable systems are well suited for industrial applications, when the common approaches for individual drives do not fulfil the requirements.

However, frequency response methods are not suitable for nonlinear systems in the first place. This is the case, for example, if an orthogonal axis with a movable mass is added to the gantry stage. In this case, the characteristics of the system change with the feed drive position. If the variation of the system is negligible for a small change of the position, the analysis can be done for different working points. This aspect will be part of future investigations.

\section{ACKNOWLEDGMENTS}

This research was supported by a German Research Foundation (DFG) grant, received within the research project "Application potential of articulated coupled drive and guide elements for increase of movement dynamics and accuracy" (IH 124/6-3), which is gratefully acknowledged.

\section{REFERENCES}

[Bohn 2016] Bohn, C. and Unbehauen, H. Identifikation dynamischer Systeme. Wiesbaden: Springer Vieweg, 2016. ISBN: 9783834821973

[Doyle 1981] Doyle, J. and Stein, G. Multivariable Feedback Design: Concepts for a Classical/Modern Synthesis. Transactions on Automatic Control, February 1981, Vol. 26, No. 1, 4-16, ISSN: 00189286
[Gordon 2012] Gordon, D. J. and Erkorkmaz, K. Precision control of a T-type gantry using sensor/actuator averaging and active vibration damping. Precision Engineering, April 2012, Vol. 36, No. 2, 299-314

[Howell 2013] Howell, L. L. et al. Handbook of Compliant Mechanisms. Chichester: John Wiley \& Sons, 2013. ISBN 9781118516515

[Hu 2010] Hu, C. et al. Coordinated Adaptive Robust Contouring Control of an Industrial Biaxial Precision Gantry With Cogging Force Compensations. Transactions on Industrial Electronics, May 2010, Vol. 57, No. 5, 17461754, ISSN 02780046

[Ishizaki 2013] Ishizaki, K. et al. Cross Coupling Controller for Accurate Motion Synchronization of Dual Servo Systems. International Journal of Automation Technology, June 2013, Vol. 7, No. 5, 514-522

[Kang 2012] Kang, D. and Gweon, D. Development of flexure based 6-degrees of freedom parallel nanopositioning system with large displacement. Review of Scientific Instruments, March 2012, Vol. 83, No. 3, 035003 [Kim 2005] Kim, D. et al. Optimal design of a flexure hingebased $X Y Z$ atomic force microscopy scanner for minimizing Abbe errors. Review of Scientific Instruments, June 2005, Vol. 76, No. 7, 073706

[Li 2015] Li, C. et al. Dual drive system modeling and analysis for synchronous control of an $\mathrm{H}$-type gantry. IEEE International Conference on Advanced Intelligent Mechatronics (AIM), Busan, July 2015, IEEE, ISSN: 21596247

[Ma 2019] Ma, J. et al. Robust Decentralized Controller Synthesis in Flexure-Linked H-Gantry by Iterative Linear Programming. Transactions on Industrial Informatics, March 2019, Vol. 15, No. 3, 1698-1708, ISSN: 15513203

[Peukert 2019] Peukert, C. et al. Modal-Space Control of a Linear Motor-Driven Gantry System. MM Science, 2019 , Vol. 12, No. 4, 3285-3292

[Poehlmann 2020] Poehlmann, P. et al. Compliant Joints for the Improvement of the Dynamic Behaviour of a Gantry Stage with Direct Drives. Journal of Machine Engineering, 2020, Vol. 20, No. 3, 17-29, ISSN 1895-7595

[Ma 2019] Ma, J. et al. Robust Decentralized Controller Synthesis in Flexure-Linked $\mathrm{H}$-Gantry by Iterative Linear Programming. Transactions on Industrial Informatics, March 2019, Vol. 15, No. 3, 1698-1708, ISSN: 15513203

[MacFarlane 1977] MacFarlane, A. G. J. and Kouvaritakis, B. A design technique for linear multivariable feedback systems. International Journal of Control, 2020, Vol. 25, No. $6,837-874$

[Skogestad 2005] Skogestad, S. and Postlethwaite I. Multivariable Feedback Control: Analysis and Design. 2. ed. Chichester: John Wiley \& Sons, 2005. ISBN 9780470011683

[Tian 2010] Tian, Y. et al. Design and dynamics of a 3-DOF flexure-based parallel mechanism for micro/nano manipulation. Microelectronic Engineering, February 2010 , Vol. 87 , No. 2, 230-241 